\title{
Beyond DNA origami: the unfolding prospects of nucleic acid nanotechnology
}

\author{
Nicole Michelotti, ${ }^{1}$ Alexander Johnson-Buck, ${ }^{2}$ Anthony J. Manzo, ${ }^{2}$ \\ and Nils G. Walter ${ }^{2, *}$
}

Nucleic acid nanotechnology exploits the programmable molecular recognition properties of natural and synthetic nucleic acids to assemble structures with nanometer-scale precision. In 2006, DNA origami transformed the field by providing a versatile platform for self-assembly of arbitrary shapes from one long DNA strand held in place by hundreds of short, site-specific (spatially addressable) DNA 'staples'. This revolutionary approach has led to the creation of a multitude of two-dimensional and three-dimensional scaffolds that form the basis for functional nanodevices. Not limited to nucleic acids, these nanodevices can incorporate other structural and functional materials, such as proteins and nanoparticles, making them broadly useful for current and future applications in emerging fields such as nanomedicine, nanoelectronics, and alternative energy. (c) 2011 Wiley Periodicals, Inc.

How to cite this article:

WIREs Nanomed Nanobiotechnol 2012, 4:139-152. doi: 10.1002/wnan.170

\section{INTRODUCTION}

Tucleic acid nanotechnology has been utilized 1 by nature for billions of years. ${ }^{1,2}$ DNA in particular is chemically inert enough to reliably store genetic information over even millions of years. ${ }^{3}$ Packaged in genomes, DNA is expressed in a regulated fashion with assistance from its more rapidly hydrolyzed structural analog, RNA, as well as protein factors. ${ }^{2,4,5}$ Complementarily, the enhanced functionality of RNA is exploited in many biological nanomachines, such as the ribosome ${ }^{6}$ and the spliceosome, ${ }^{7,8}$ for the 'data processing' that accompanies gene expression. Nature also uses the specificity of base pairing by nucleic acids for database maintenance and readout via the modulation of gene expression by noncoding RNAs (ncRNAs). ${ }^{4,9}$

\footnotetext{
*Correspondence to: nwalter@umich.edu

${ }^{1}$ Department of Physics, University of Michigan, Ann Arbor, MI, USA

${ }^{2}$ Department of Chemistry, University of Michigan, Ann Arbor, MI, USA
}

Inspired by nature, researchers over the past four decades have explored nucleic acids as convenient building blocks to assemble novel nanodevices. ${ }^{1,10}$ Because they are composed of only four different chemical building blocks and follow relatively simple, yet highly specific and thus predictable organizational base pairing rules at the molecular scale, nucleic acids are the preferred biological material for the design of structures with nanometer precision when compared with other candidates such as proteins. Short [typically $<50$ nucleotides (nt) for RNA and $<100 \mathrm{nt}$ for DNA] specific sequences of the building blocks can be chemically synthesized at low cost, whereas longer DNA strands of predefined sequence are provided by nature in the form of genomic DNA. A combination of these two sources led to the advent of the DNA origami method in $2006,{ }^{11}$ which dramatically accelerated progress in nucleic acid nanotechnology by further increasing the simplicity, precision, and fidelity of the design principles available for generating spatially addressable nanoscale structures (Box 1). Numerous DNA origami-based nucleic acid nanodevices and nanomaterials have since been 
constructed $^{12-16}$ with great potential for a multitude of useful applications and with abundant prospects for innovation.

\section{BOX 1: DNA ORIGAMI}

The invention of scaffolded DNA origami was a milestone in the advancement of nucleic acid nanotechnology. Developed by Paul Rothemund, DNA origami is the assisted folding of one long ssDNA 'scaffold' strand from a bacterial phage genome consisting of 7249 nucleotides into a predetermined shape by $\sim 200$ typically 32-nt-long ssDNA 'staple' strands containing sequences that are complementary to specific regions of the scaffold strand. Two or more nonadjacent segments of the scaffold strand are brought together and held in place by hybridization to different portions of the same staple oligonucleotide in aggregate (Figure 3(a)), enabling the creation of arbitrary shapes based solely on the staple sequences (Figure 3(b) and (c)). The staples may be extended at their $5^{\prime}$ ends to make them addressable for patterning with a resolution of $6 \mathrm{~nm}$ on the assembled origami, imposed by the interstaple distance on the assembled DNA duplexes of the origami ${ }^{11}$ (Figure $3(d)$ ).

While building on previous approaches to self-assembly of DNA nanostructures, the DNA origami technique has multiple advantages: (1) It accomplishes highly specific topologies with a spatially addressable resolution comparable to that accomplished by surface manipulation using atomic force microscopy (AFM) or scanning tunneling nicroscopy (STM). (2) The well-formed, redundant self-assembly is relatively insensitive to varying stoichiometric ratios of the staples, eliminating the need for intermittent purification steps and resulting in higher yields. (3) Multiple nanostructures may be obtained simultaneously and possibly further assembled into larger structures with high fidelity. (4) Creating arbitrary shapes that are largely unrestricted by symmetry considerations is relatively straightforward. ${ }^{11}$

The DNA origami method has so far provided a scaffold for many applications including forming the track for molecular nanorobots, ${ }^{47}$ constructing ordered molecular assembly lines, ${ }^{48,49}$ and assembling components for nanoelectronic circuits. ${ }^{50,51}$ The scope and limitations of these applications are enumerated throughout.

\section{THE BUILDING MATERIALS: NATURAL NUCLEIC ACIDS}

Nucleic acids are linear biopolymers found in all organisms, as well as many viruses, as they are the means by which genetic information is stored, transferred, and regulated. Their monomeric building blocks (nucleotides) each consist of three moieties (Figure $1(\mathrm{a})-(\mathrm{c}))$ : a nucleobase, a five-carbon sugar, and a phosphate group. The phosphate group enables the formation of a phosphodiester bond between the sugars of adjacent nucleotides, creating a polymer known as a single-stranded nucleic acid. Complementary nucleobases can form hydrogen bonds which, along with stacking interactions between adjacent base pairs, result in two fully complementary polymers hybridizing to form a relatively rigid antiparallel double-stranded helix ${ }^{17}$ (Figure 1(d)). This famous Watson-Crick duplex has an average dimension (rise) of $0.34 \mathrm{~nm}$ (for canonical B-form DNA) between the base pairs along the helical axis and a diameter of $2 \mathrm{~nm}$, ideally suited to engineer nanotechnological structures.

Nature has optimized two different types of nucleic acids through evolution: DNA and RNA, which both consist of four nucleotide building blocks that tend to pair in a predictable Watson-Crick fashion (Figure 1(d)). The guanine-cytosine (G:C) base pair forms three hydrogen bonds, stronger than the two hydrogen bonds of the adenosine-thymine (A:T) pair, used in DNA, or the adenosine-uracil (A:U) pair, used in RNA. The primary chemical difference between DNA and RNA is their sugars (Figure 1(a)); ribose, the sugar found in RNA, contains an extra 2'-hydroxyl group not present in deoxyribose, the sugar found in DNA, causing the backbone of RNA to hydrolyze $10^{8}$ - to $10^{10}$ times more quickly than that of DNA $^{18}$ (Figure 1(a)). The A-form RNA duplex, however, is more thermally stable than the B-form DNA duplex, even though RNA is predominantly found single-stranded in nature and forms mostly intramolecular helices. ${ }^{19}$ These structural differences between DNA and RNA are functionally exploited by nature.

\section{DNA}

While the most notable purpose of DNA is storing and maintaining the genetic blueprint of an organism, DNA performs other structural roles in the cell as well. For instance, the guanine-rich singlestranded telomeres at the ends of chromosomes can 
(a)

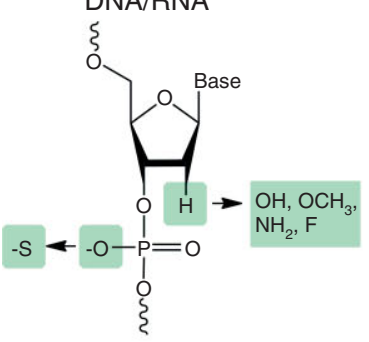

(b)

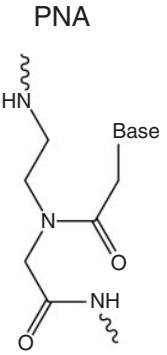

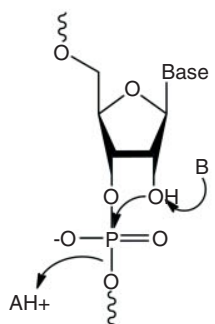

(c)

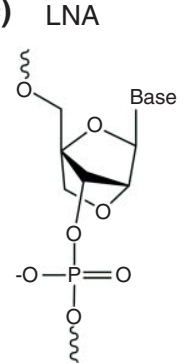

(d)

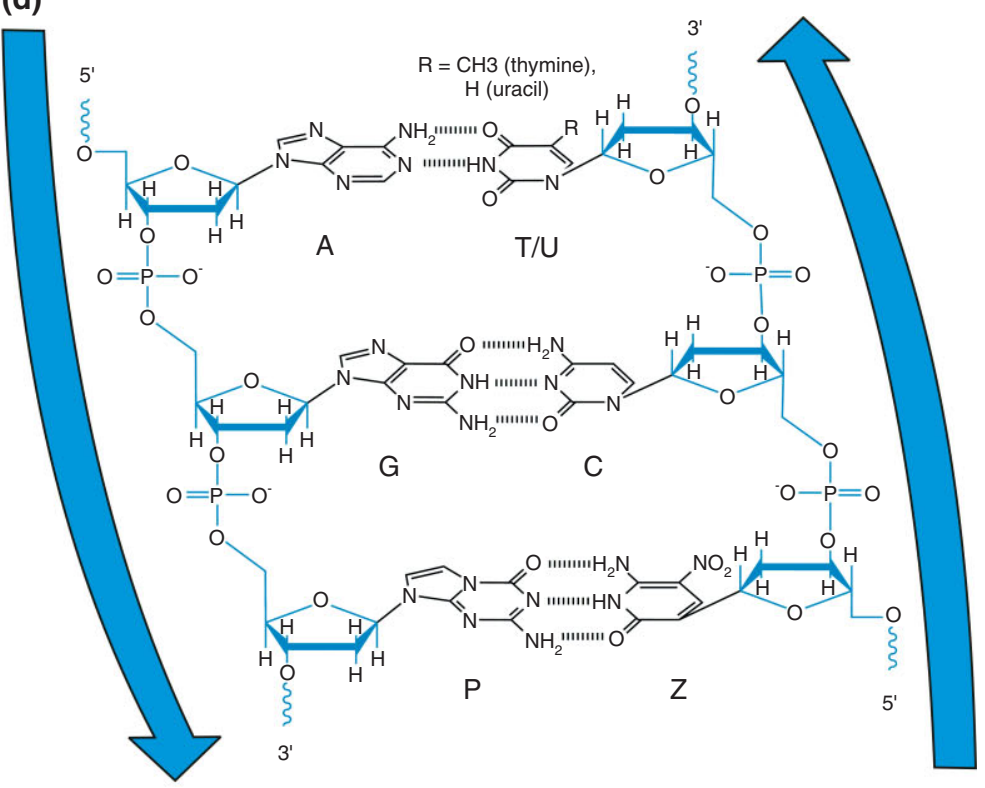

FIG URE 1 | Chemical structures of nucleic acids. Nucleotide backbone structures for (a) DNA (left), RNA (right), (b) PNA, and (c) LNA. The right side of panel (a) also indicates the backbone hydrolysis reaction of RNA, where ' $A H^{+'}$ and ' $B$ ' are an acid and base catalyst, respectively. (d) Structure of a double-stranded nucleic acid incorporating the four principal natural nucleobases $A, T / U, G$, and $C$, as well as the artificial $P: Z$ base pair.

form topologically more complex quadruplex structures built around square stacks of four guanine bases stabilized by a chelated metal ion (preferably $\mathrm{K}^{+}$) at their center. G-quadruplexes thus play important roles in the maintenance of linear eukaryotic genomes and possibly in gene expression regulation. ${ }^{20}$ Similarly, so-called Holliday four-way junctions of two entwined DNA duplexes play critical roles in DNA recombination. ${ }^{21}$ Forming and resolving such higherorder structures often requires the aid of external factors such as proteins. For example, complexes of histone proteins facilitate the compact folding of $\sim 1$ $\mathrm{m}$ of DNA into chromosomes that fit inside a $\sim 6$ $\mu \mathrm{m}$-wide nucleus while still remaining accessible for gene expression. ${ }^{22,23}$ In cases where decreased stability would be most beneficial, a lower melting temperature may be achieved by increasing the ratio of A:T to G:C base pairs, as is desirable for promoter regions in DNA (regions that need to melt for an RNA polymerase to initiate gene expression ${ }^{24}$ ).

\section{RNA}

In the central dogma of molecular biology, the portrayed function of RNA is as the messenger between DNA and protein, carrying the blueprints for protein formation from the DNA genome so that they may be translated by the ribosome into proteins. ${ }^{25}$ However, over the past few decades, the pervasiveness and importance of RNA that does not code for proteins have been increasingly appreciated. Typically, these ncRNAs directly or indirectly regulate or mediate gene expression at the transcriptional or translational level, making them candidates for therapeutic applications. ${ }^{4,9,26}$ Some ncRNAs simply make use of molecular recognition through base pairing, such as in the RNA interference pathway. ${ }^{26}$ Others exploit conformational dynamics of RNA. ${ }^{27}$ For example, riboswitches regulate gene expression via the conformational change induced by the RNA binding a smallmolecule metabolite or second messenger. ${ }^{28}$ Still other ncRNAs promote catalysis, such as in peptide bond formation within the ribosome ${ }^{6,29}$ or cleavage of RNA via hydrolysis of the sugar-phosphate backbone ${ }^{30}$; the activity of each of these RNA enzymes (ribozymes) is dictated by its tertiary structure. While these activities are currently difficult to engineer from scratch due to the large number (hundreds to thousands) of nucleotides required and our limited understanding of the underlying mechanisms, these functional, structurally complex ncRNAs have already been optimized by nature as active components in cellular machines.

\section{THE BUILDING MATERIALS: MODIFIED NUCLEIC ACIDS}

Nucleic acids undergo chemical base modifications in vivo, impacting their cellular functions. For 
example, cytosine methylation prevents DNA from being digested by methylation-sensitive restriction enzymes in bacteria, ${ }^{31}$ and in many eukaryotic organisms, it is involved in the epigenetic regulation of gene expression. ${ }^{32}$ In an analogous fashion, researchers have synthetically modified nucleotides to increase thermodynamic and chemical stability as well as specificity of interactions. For instance, locked nucleic acids (LNA) are engineered with an extra methylene bridge between the $2^{\prime}$-oxygen and $4^{\prime}$-carbon of ribose, 'locking' the ribose in the $3^{\prime}$ endo conformation (Figure 1(c)), which leads to a particularly stable A-form helix with enhanced base stacking and backbone preorganization as well as significantly increased melting temperature, specificity of base pairing, and nuclease resistance. ${ }^{33,34}$ Other chemical modifications of the nucleic acid backbone commonly used for enhancing intracellular stability while still often supporting biological function of an ncRNA include 2'-O-methyl, 2'-amino, 2'-fluoro, and phosphorothioate substitutions ${ }^{35}$ (Figure 1(a)). As a more drastic modification, peptide nucleic acids (PNA) have an uncharged backbone of $N$ (2-aminoethyl)-glycine units joined by a peptide bond, resulting in higher thermal stability between PNA/DNA strands than corresponding DNA/DNA strands $^{36}$ (Figure 1(b)). The uncharged nature and nuclease resistance of PNA make it an appealing option for nanodevices ${ }^{37}$ and $2 \mathrm{D}$ nucleic acid arrays. ${ }^{38}$

Not only can the nucleic acid backbone be modified but also the nucleobases themselves. A particularly far-reaching example is the artificial expansion of the genetic alphabet by an additional, differently hydrogen bonded base pair such as Z:P (or 6-amino-5-nitro-3-(1'- $\beta$-D-2'-deoxyribo furano syl)-2(1H)-pyridone paired with 2 -amino- 8 - $\left(1^{\prime}-\beta\right.$-D$2^{\prime}$-deoxyribofuranosyl)-imidazo[1,2-a]-1,3,5-triazin-4 $(8 \mathrm{H})$-one, respectively; Figure $1(\mathrm{~d}))$, which increases hybridization specificity while still allowing for specific recognition by DNA-binding enzymes. ${ }^{39}$

Whereas the improved thermal and chemical stability often found in modified nucleic acids will prove useful for nanostructures, incorporation is nontrivial; nanostructure design is heavily dependent on helical twist, which is typically different between modified and unmodified nucleic acids. ${ }^{10}$ This hurdle will, however, be overcome with improved and expanded design rules. In addition, these materials can already be used to site-specifically decorate DNA scaffolds since they form canonical Watson-Crick base pairs.

\section{THE PRESENT STATE OF NUCLEIC ACID NANOTECHNOLOGY}

By employing the knowledge acquired from observing nature and building upon it, nanostructures not found in nature have been engineered that encompass a broad range of design objectives. The following are examples of successful nanostructures grouped according to their purpose.

\section{Scaffolding}

Nucleic acid scaffolds are nanostructures that may be functionalized for ordering and arraying materials with nanometer precision. Scaffolds were originally formed by combining different short double-stranded DNA (dsDNA) domains joined in a programmable fashion using single-stranded DNA (ssDNA) overhangs known as 'sticky ends'. ${ }^{40}$ While the stiffness of dsDNA makes it a suitable raw material for the edges of stable two- and three-dimensional structures, the vertices of such structures remain flexible, resulting in a range of angles between domains. ${ }^{40}$ Inspired by naturally occurring Holliday junctions, ${ }^{21,41}$ more rigid structures were accomplished using reciprocal exchange to generate double ${ }^{42}$ and triple crossover motifs $^{43}$ (Figure 2). As an alternative to combining multiple domains, which results in variable yields of assembled structures without defined boundaries, increased yield and stability are accomplished using Rothemund's DNA origami method (Box 1 and Figure 3), leading to more sophisticated 2D and 3D

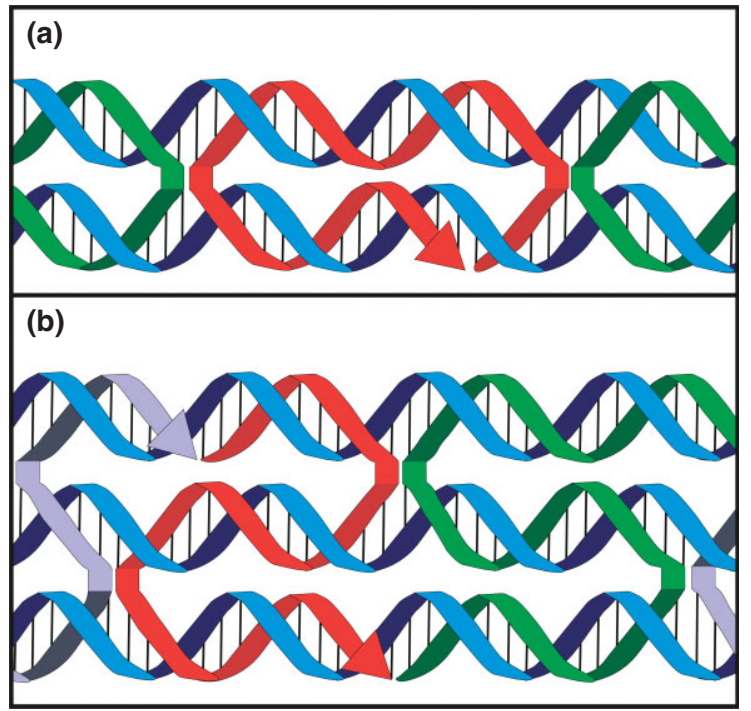

FIG URE 2 | DNA crossover motifs. Examples for (a) double and (b) triple crossover motifs. The red strands show how the two and three helices, respectively, are linked. 

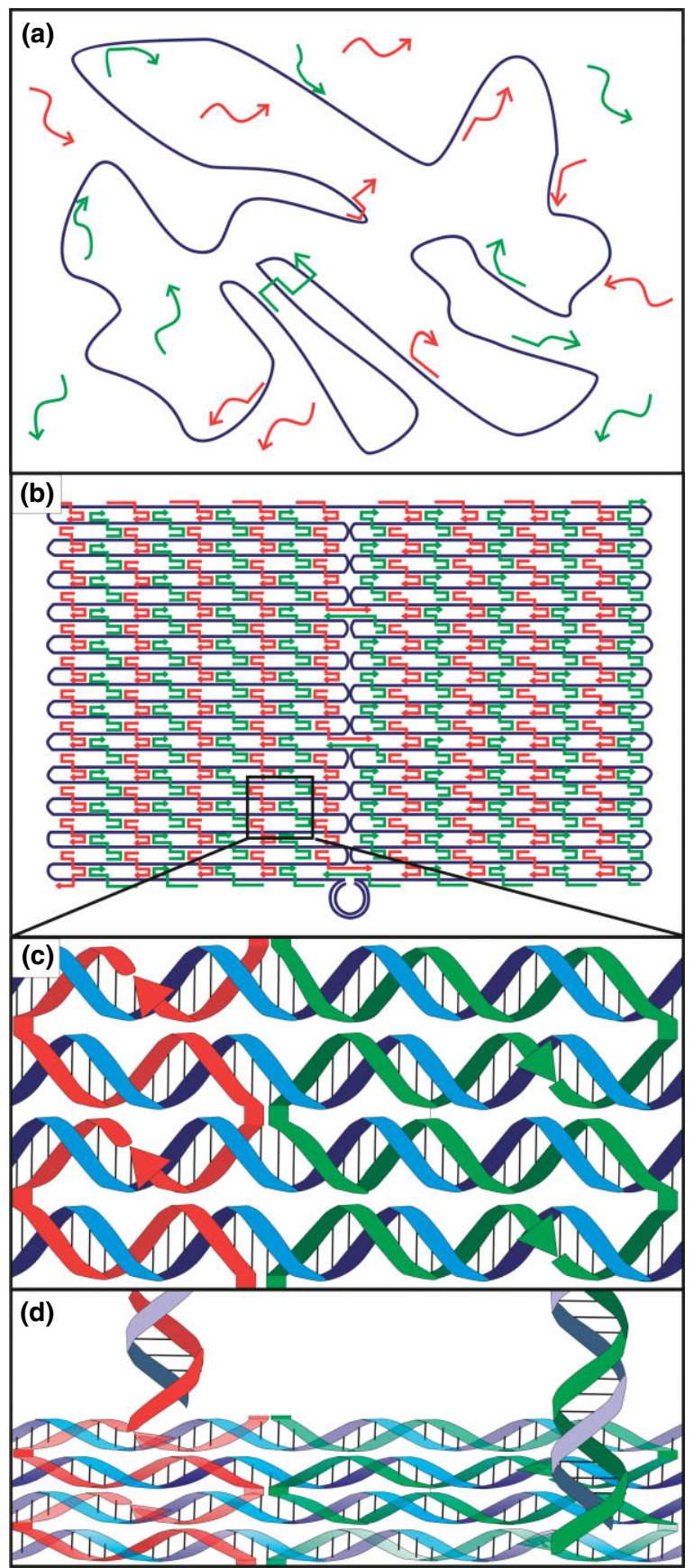

FIG URE 3 | The DNA origami method. (a) The single-stranded DNA 'scaffold' strand (purple) is folded and held in place by specifically hybridizing 'staple' strands (red and green). (b) Resultant rectangular origami tile once all the staples have bound to the scaffold. (c) Triple crossover motifs demonstrated by the staples interacting with the scaffold. (d) 5' ends of the staples are extended to create overhangs, which then can be decorated with partially complementary oligonucleotides (purple).

structures ranging from smiley faces ${ }^{11}$ to octahedra ${ }^{44}$ and nanoflasks ${ }^{45}$ that can be concatenated into larger structures. ${ }^{11,46}$

\section{Dynamic Devices Constructed on Nucleic Acid Scaffolds}

To add functionality to the scaffolds, DNA, RNA, and protein have further been used to construct dynamic devices serving as proofs of principle for directed transport and nanoscale assembly factories. Molecular walkers analogous to the motor proteins myosin, kinesin, and dynein have been constructed completely or primarily from DNA and have attained increasing levels of autonomy as well as responsiveness to external instruction ${ }^{52,53}$ (Figure 4(a)). Some such transporters are capable of dozens to potentially thousands of directed steps and have been shown to walk processively along tracks on DNA origami. ${ }^{47,52}$ Directed walkers have also aided in the combinatorial assembly of different sized gold nanoparticles ${ }^{48}$ or the multistep synthesis of a small organic compound, ${ }^{49}$ in the former case aided by two-state DNA conformational switches (Figure 4(b)). Coupling such devices with DNA computing, ${ }^{54}$ either on the scaffold or in solution, may further improve the complexity and range of responses to environmental stimuli or instructions.

In addition to the above devices made primarily of nucleic acids, several groups have sought to augment the functional repertoire of DNA and RNA nanotechnology through conjugation to other nanomaterials, using self-assembled DNA structures as a scaffold on which to position non-nucleicacid components that impart some new catalytic, electronic, photonic, or structural functionality. For instance, placing the metabolically linked enzymes luciferase and $\mathrm{NAD}(\mathrm{P}) \mathrm{H}$-dependent FMN oxidoreductase (NFOR) in close proximity on a DNA template led to more efficient recycling of their common $\mathrm{FMN} / \mathrm{FMNH}_{2}$ cofactor and an overall enhancement of the catalyzed reaction rates, mimicking the strategy of substrate channeling often employed by nature. ${ }^{58}$ DNA origami has been used to create junctions of carbon nanotubes with precisely defined geometry, ${ }^{50,51}$ in one case behaving like a nanoscale field-effect transistor $^{50}$ (Figure 4(c)). Furthermore, DNA templates have directed the assembly of chromophores such as organic dyes and quantum dots for the directed transfer of photonic energy through fluorescence resonance energy transfer (FRET), ${ }^{55,59,60}$ with the prospect of developing photonic circuits or artificial photosynthetic antenna complexes (Figure $4(\mathrm{~d})$ ). Conjugating DNA to small organic molecules or polymers has yielded materials with interesting thermal properties, such as solid DNA that only melts at $95^{\circ} \mathrm{C} .{ }^{61}$ Hybrids of nucleic acids and synthetic polymers show promise for drug delivery and diagnostics. ${ }^{62}$ Finally, 
(a)
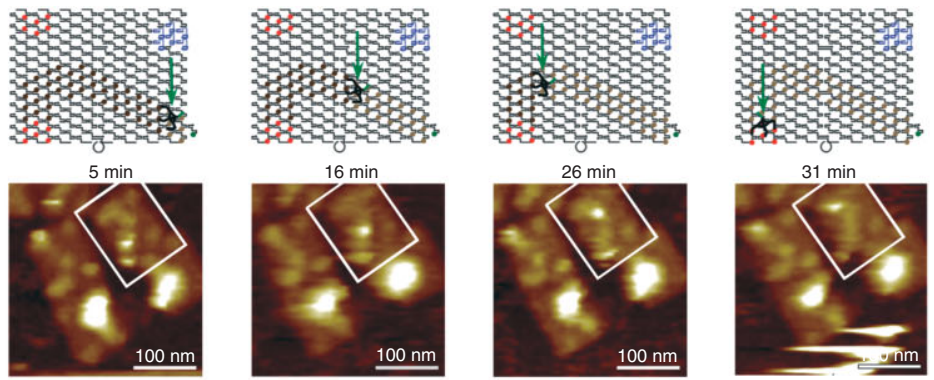

(b)
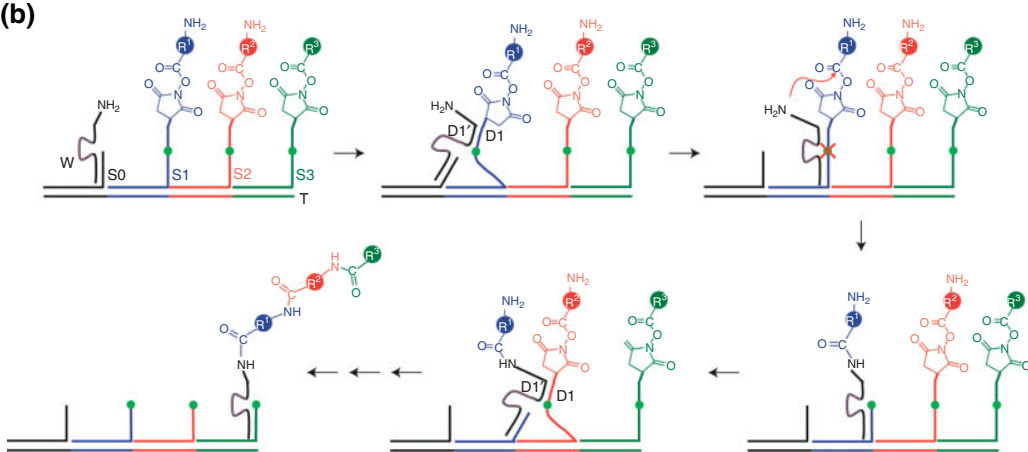

(c)

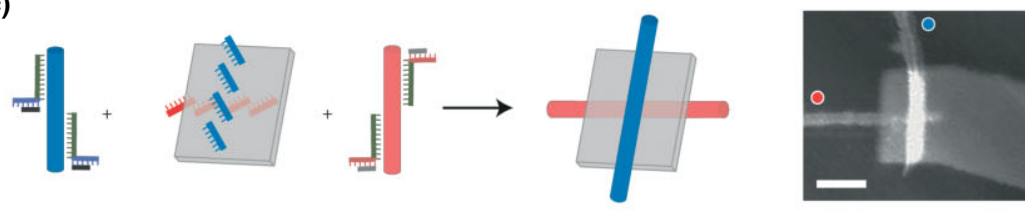

(d)

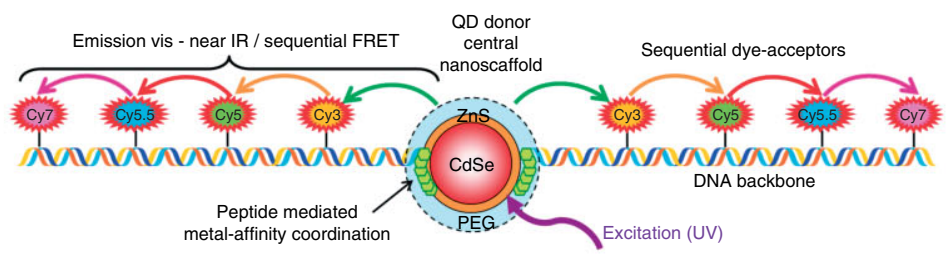

(e)

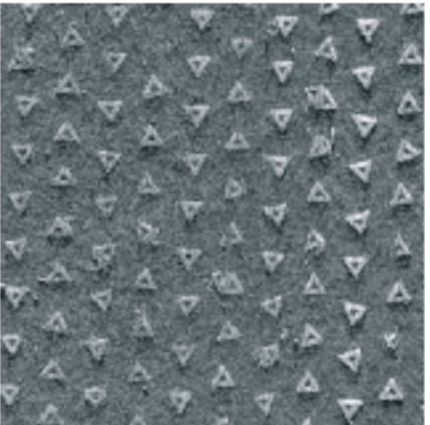

(f)

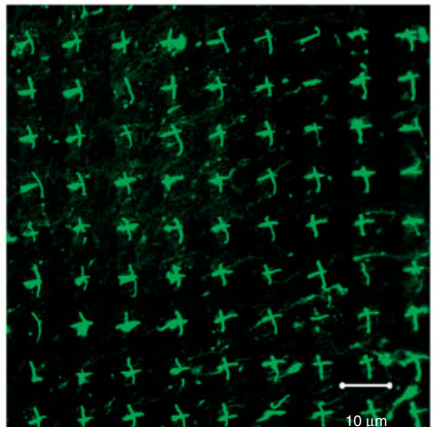

proteins may also be employed as integral structural components of nucleic acid nanoassemblies. For instance, assemblies shaped like equilateral triangles were formed from an RNA-binding protein and an RNA strand bearing the appropriate binding motif. ${ }^{63}$

\section{Combining Bottom-up with Top-down Techniques}

So far, we have described nanoassemblies built using bottom-up techniques (i.e., the creation of more complicated structures self-assembled from 
fundamental units). While these techniques yield highly resolved structures that may be generated in parallel, they are so far still limited in their dimensions, making them unappealing for applications where truly large-scale patterning is required. By contrast, most current technology outside of nanotechnology uses top-down techniques, namely, the ordered assembly of components by externally controlled means. For example, many lithographic top-down fabrication methods are able to serially generate structures with features typically on the order of microns (e.g., for silicon-based computer chips) but at great capital expense for equipment. By combining topdown and bottom-up techniques, a more affordable large-scale $(>1 \mathrm{~mm})$ patterned array with nanometer addressability may be accomplished. ${ }^{64}$ Electron beam ${ }^{65}$ and soft ${ }^{57}$ lithography techniques have been used to pattern DNA nanostructures across a surface with spacings of $\sim 300 \mathrm{~nm}$ and $\sim 5 \mu \mathrm{m}$, respectively (Figure 4(e) and (f)).

\section{ENVISIONING FUTURE APPLICATIONS}

We envision that the basic materials and concepts of nucleic acid nanotechnology outlined so far will further be recombined to create devices capable of accomplishing an ever wider variety of tasks, particularly in the following fields (Figure 5).

\section{Molecular Computing}

Modern-day computers are capable of performing incredibly complex calculations with impressive speeds. At the computer's core, these complex calculations are made possible by large series of binary digital circuits incorporating logic gates. Analogously, nucleic acids can behave as logic gates. For instance, the output 'true' for an AND logic gate can be represented by a deoxyribozyme cleaving its substrate if and only if both DNA strand A and DNA strand $\mathrm{B}$ are present in solution ${ }^{66}$ (Figure 6). Using variations on this basic concept, nucleic acids have completed tasks with a range of complexities from competitively playing tic-tac-toe ${ }^{67}$ to mimicking field programmable gate arrays ${ }^{68}$ and neural networks. ${ }^{69}$ Nucleic-acid-based logic gates inherently function based on thermodynamic mass action and chemical kinetics laws (i.e., probabilistic behavior), rather than the deterministic, digital on-off switches of silicon-based computers. Computing by nucleic acid nanotechnology therefore will more closely mimic the 'fuzzy logic' and feedback loops of ant colonies and the human brain, with corresponding advantages in adaptability and complex pattern analysis. ${ }^{70}$ As such, it will likely find ample application in both probing and interfacing with biology.

\section{Plasmonics}

When metallic nanoparticles interact with visible electromagnetic waves, their conduction-band electrons are excited, resulting in a phenomenon known as plasmon resonance. ${ }^{71,72}$ These nanoparticles placed in close proximity $(<2.5$ times the diameter of the nanoparticle) undergo strong near-field coupling with field enhancements. ${ }^{73}$ However, the optimal field enhancement is dependent on the geometry of the nanoparticle array, requiring precise nanoparticle placement and spacing. ${ }^{71,73}$ To this end, gold nanoparticles have been assembled on DNA origami triangles. ${ }^{56,74}$ While DNA origami tiles are advantageous in their rigidity and addressability, they are limited in length $(\sim 100 \mathrm{~nm})$ unless concatenated, in turn limiting the propagation of the plasmon resonance signal. In the future, the longer scaffolds, such as the nanopeapod ${ }^{75}$ or DNA origami nanotube ${ }^{76}$ with lengths on the order of tens of microns, will be paired with top-down microfabrication techniques to construct larger nanoparticle arrays with precision and spacing on the order of tens of nanometers. Applications will likely include communication systems that merge electronics and photonics (optics) at the nanoscale. ${ }^{71}$

\section{Biosensors}

Biosensors are small, self-contained devices that detect and report the concentration of an analyte through physical interaction. ${ }^{77}$ Several types of biosensors have been constructed from nucleic acids (e.g., aptazyme-based ${ }^{78}$ or $\mathrm{pH}$-sensitive ${ }^{79}$ ), the most common being optical biosensors in which the hybridization between the analyte and its complementary capture strand immobilized on the surface results in a change in fluorescent signal. ${ }^{77}$ To be effective, such a technique requires a highly specific interaction between the capture strand and the analyte and low yield of false-positive results. Increased specificity has already been accomplished by incorporating synthetic nucleic acids, ${ }^{80,81}$ although there is still room for improvement, for example, with the further development of modified nucleotides. One of the causes of false-positive signals is crosstalk between neighboring capture strands. ${ }^{82}$ The spacing between neighboring biosensor units may be determined with nanometer precision by using a DNA origami scaffold, enabling a high density of capture strands on the surface while limiting their interactions. Such scaffold techniques have recently 


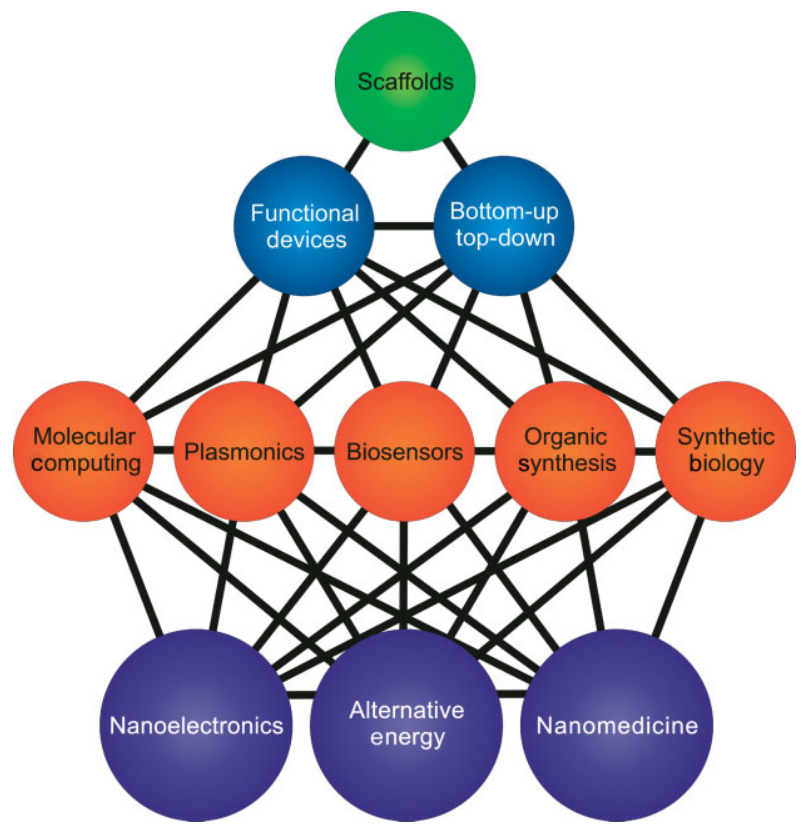

FIG URE 5 | Hierarchy of areas impacted by DNA origami technology. The foundational DNA scaffolds (green) are used to create devices (blue) for a broad variety of applications (orange) that can be combined to enhance numerous emerging interdisciplinary fields (purple). Note that the nucleic acid nanotechnology field will not be limited by our current vision.

been utilized to detect analyte concentrations as low as $200 \mathrm{pM}$, and it was found that the hybridization sensitivity was dependent on probe position. ${ }^{83}$ In addition, precisely arranged biosensor arrays with enhanced and possibly synergistic detection properties for complex samples are now within grasp.

\section{Organic Synthesis}

Unlike conventional organic synthesis, which is performed one step at a time with pure reagents, the cell is capable of catalyzing thousands of reactions simultaneously in the same vessel with extremely high specificity and efficiency. DNA scaffolds and devices may provide a way to emulate this approach by virtue of their programmable nanoscale structure and ability to spatially organize catalysts and reagents. DNA origami, in combination with singlestranded overhangs, conformational DNA switches, and/or DNA walkers, have been used for multistep organic synthesis, ${ }^{49}$ programmable assembly of dendrimeric oligomers, ${ }^{84}$ and dynamically controlled combinatorial assembly of gold nanoparticles. ${ }^{48}$ Since DNA origami are currently expensive to produce in large quantities, practical applications may be restricted in the short term to small-scale syntheses, those that must be performed under mild conditions or those that are not feasible using conventional synthetic chemistry-for example, when high-resolution control of reactant positioning (different than that afforded by surface or metal ion coordination chemistry) ${ }^{84}$ is required. Such syntheses are abundant in nature and exemplified by the assembly lines of multidomain polyketide synthases that generate a large number of bioactive compounds. ${ }^{85}$ Another exciting prospect enabled by coupling DNA origami to other dynamic devices is the ability to make chemical reactions or noncovalent molecular assembly responsive to environmental cues. $^{48}$ For instance, signals from a host organism might provide cues for a smart molecular 'assembly line' to synthesize one of many possible products depending on intracellular conditions at a particular time.

\section{Synthetic Biology}

Synthetic biology is concerned with engineering natural or artificial biological systems to better understand biological phenomena or for practical uses such as food, drug, fuel production, or novel therapeutic approaches. ${ }^{86,87}$ While most synthetic biology has made use of pre-existing pathways by altering them for a particular purpose or inserting them into a different organism (top-down approach), ${ }^{86}$ it may become possible to construct entirely novel artificial organisms ${ }^{88}$ and pathways to allow for more predictable and tractable performance (bottom-up approach). ${ }^{86}$ DNA origami and similar scaffolds could contribute to such systems by allowing for spatial and chemical coupling of related enzymes in a manner analogous to natural multienzyme complexes, a concept that has recently been demonstrated on simpler DNA templates ${ }^{89}$ in vitro and in highly concatenated RNA scaffolds in bacteria. (The latter demonstrates one clear advantage of RNA for many synthetic biology applications: it is readily transcribed from a plasmid inserted into the host organism.) Three-dimensional nucleic acid scaffolds could also serve to physically compartmentalize artificial biochemical reaction networks like natural organelles, since they can be engineered to assemble with high efficiency into vessels of arbitrary shape with well-defined dimensions. ${ }^{45,90,91}$ Furthermore, due to the relative ease of engineering nucleic-acid-based reaction networks ${ }^{54}$ and the existence of numerous elementary components such as aptamers, ribozymes, and riboswitches, some of the first artificial biological systems or organisms may have nucleic acids as integral components of their information networks. Although true bottom-up synthetic biology remains 


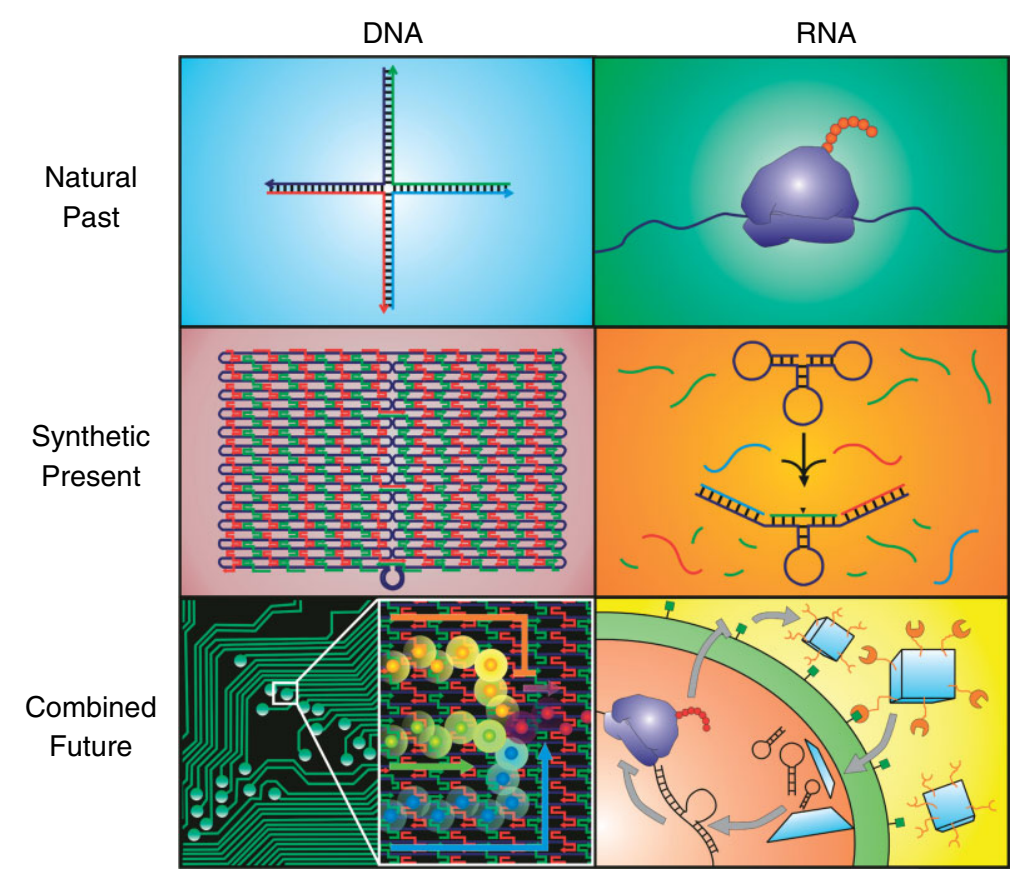

FIG URE 6 | Progression in DNA and RNA nanotechnology. In nature, DNA forms structures such as the Holliday junction (field: DNA, Natural Past), which has inspired scientists to create more complex structures such as the rectangular DNA origami tile (DNA, Synthetic Present). In the future, such tiles may be used in fields including nanoelectronics as a scaffold for plasmonic circuit components to generate circuits that mimic neuron behavior (DNA, Combined Future). As the simple example in the figure depicts, a new circuit connection (purple) may be strengthened by repeated cooperative stimulation from excitatory pathways (green and blue) and hindered by the stimulation from an inhibitory pathway (orange). In nature, RNA plays a catalytic role in peptide bond formation by the ribosome, arguably the most important enzyme on earth (RNA, Natural Past). The catalytic and exquisite molecular recognition activity of RNA is exploited in (deoxy)ribozyme computing (RNA, Synthetic Present), which may be used in the future for complex therapeutic nanomedicine applications. For instance, a drug carrier, specifically delivered to a diseased cell through endocytosis triggered by binding to a protein on the cell surface, opens after entering the cell to release the drug (RNA, Combined Future). The contents of the drug carrier include a microRNA mimic that causes repression of a specific protein that otherwise would inhibit the surface marker, resulting in a cell that becomes more receptive to the drug carriers.

an elusive prospect for now, nucleic acid scaffolds and devices are beginning to serve as a useful means to perturb biochemical networks. ${ }^{92-94}$ Since DNA origami are stable in cells ${ }^{93,94}$ and cell lysate, ${ }^{95}$ even more complex structures may soon find similar uses in interfacing with and programming of natural biological pathways.

\section{EMERGENT INTERDISCIPLINARY FIELDS}

Beyond the horizon of advantageous applications in more or less well-established fields, it is illuminating to explore the current state of some emerging interdisciplinary fields (Figure 5) to better understand how they are expected to benefit from future advancements in nucleic acid nanotechnology.

\section{Nanoelectronics}

There exists a strong effort to make more highly sophisticated, conveniently sized electronic devices, requiring the further minimization of electronic components. Currently, top-down-produced assemblies are limited by the size of nanowires and use of lithographic techniques to tens of nanometers in spatial resolution. The wires are also limited in the flux of digital information they are able to transport when compared to optical digitization techniques such as optical fibers. ${ }^{96}$ Exploiting plasmonic properties between precisely placed nanoparticles is a plausible alternative. Positioned using nucleic acid scaffolds, such an architecture would not only allow for signal enhancement of the propagated information but also, theoretically, the nanoparticles could behave as various, reconfigurable circuit components based on their materials and geometries ${ }^{97}$ (Figure 6). Integrating this approach with established lithographic techniques will lead to synergy. Scaffolded components could also serve as templates for metal deposition to generate solid-state devices with nanometer precision, although it will be necessary to develop methods for sustaining the 
template in the adverse conditions required for deposition. Arraying various bottom-up circuit elements using top-down assembling techniques may generate larger, more complex circuits.

\section{Alternative Energy}

Rising global demand for energy and concerns about the environmental impact of fossil fuel use motivate the search for viable alternative energy sources. ${ }^{98,99}$ While progress has been made toward improving alternative energy production and storage using nanomaterials, emphasis has been placed on inorganic materials rather than biomolecules. Owing to their complexity, stand-alone nucleic acid nanodevices are not likely to meet large-scale energy needs anytime soon but become more formidable when combined with living systems or inorganic materials. For example, genetically encoded nucleic acid scaffolds can be used to increase the flux through engineered biochemical pathways by colocalizing functionally related enzymes, including in the production of fuels such as hydrogen. ${ }^{100}$ Nanoelectronic and photonic devices constructed using nucleic acid scaffolds and suitable chromophores could, with sufficient improvements in efficiency, mimic photosynthetic antenna complexes for nanoscale solar energy harvesting.

\section{Nanomedicine}

Nanomedicine has been defined as the utilization of nanotechnology for medicinal purposes including diagnostic assays, therapeutic agents, and monitoring devices. ${ }^{101}$ One of the current main objectives in the field is targeted drug delivery in which a drug carrier is able to selectively deliver a drug only to diseased cells, resulting in lower drug dosages and reduced harm to healthy cells. ${ }^{101}$ Three-dimensional DNA smart materials have several features that make them appealing candidates for nanocarriers: they are biocompatible; they can selectively contain particles, fully encapsulate them, and release them when presented with a trigger; the exteriors may be decorated with targeting signals; and differing functional components may be incorporated into their design with defined spatial positioning. Current designs initiate cargo release by introducing a nucleic acid strand to the carrier's environment. ${ }^{90} \mathrm{By}$ alternatively incorporating functional strands that are sensitive to natural changes in cellular environments, such as potassium-sensitive deoxyribozymes or DNA strands that undergo conformational changes in response to $\mathrm{pH},{ }^{93}$ or an intrinsic cellular component, cargo release may be controlled intracellularly by natural means (much like intracellular genome release from a viral capsid ${ }^{102}$ ). Computational nucleic acid devices that mimic ncRNAs are being created to implement this goal by using selfcleaving deoxyribozyme hairpins that contain within their sequence a short ssDNA strand that is complementary to the portion of mRNA needing to be suppressed but that is not active unless triggered to be cleaved by disease indicators in the cellular environment. ${ }^{54,66,103}$ These devices could be integrated with the nanocarrier design to be transported only to the diseased cells. Beyond that, complex molecular 'communities' could integrate biosensors, molecular computation, and chemical synthesis to diagnose and treat illnesses at the level of individual cells. For instance, one such system might enter a cell through a carrier-mediated interaction, detect abnormal levels of a certain subset of messenger RNAs, compute the probability that a given cell is cancerous, and then decide whether to initiate apoptosis through an intrinsic cellular pathway (Figure 6). While such an application remains technically very challenging and expensive, most of the required device classes have been constructed.

DNA does have the disadvantage that it is susceptible to nucleases, potentially leading to release of cargo before triggered to do so. To overcome this challenge, modified nucleic acids may be incorporated, ${ }^{104}$ although any foreign materials proposed for therapeutic use will need to be rigorously tested for cytotoxicity and unexpected side effects. Before these devices can be used as therapeutic agents, fail-safe features (that lead, for example, to instability of the device outside of its target area) will need to be included in the design to prevent any undesired side effects.

Another primary objective of nanomedicine is the creation of point-of-care devices, which provide a convenient way of testing and diagnosing a patient immediately. Biosensors constructed with top-down techniques hold much promise for this goal. However, the currently limited sensitivity of biosensors generally requires the amplification of the analyte, typically accomplished using polymerase chain reaction, which limits the detection efficiency. ${ }^{82}$ Plasmonics may help amplify fluorescent signals, enabling a binding event to be detected even with low concentrations of the analyte. ${ }^{72}$ Integration of biosensors with nanoelectronic devices could potentially accomplish the types of analysis required to accurately diagnose a patient. 


\section{CONCLUSIONS}

Nucleic acid nanotechnology has come a long way since its inception a quarter century ago. It has advanced from purely static scaffolds to dynamic functional devices that include other natural and synthetic materials. These advances have been further aided by the recent emergence and exploitation of single molecule tools, for example, super-resolution single-molecule fluorescence microscopy, ${ }^{47,52,105,106}$ that can monitor the dynamic (bio)chemical reactions, actuation, movement (diffusion), and integrity of individual nanodevices. The resultant nanodevices show great promise for useful future applications in a broad variety of fields as sampled here. It is even more exciting to contemplate that the precise structures and functional versatility of devices such as these will spawn new applications and perhaps entire new fields that lie beyond the current bounds of our insight.

\section{REFERENCES}

1. Seeman NC. DNA in a material world. Nature 2003, 421:427-431.

2. Voet D, Voet JG. Biochemistry. 4th ed. New York: John Wiley \& Sons; 2011.

3. Paabo S, Poinar H, Serre D, Jaenicke-Despres V, Hebler J, Rohland N, Kuch M, Krause J, Vigilant L, Hofreiter M. Genetic analyses from ancient DNA. Annu Rev Genet 2004, 38:645-679.

4. Mattick JS, Taft RJ, Faulkner GJ. A global view of genomic information-moving beyond the gene and the master regulator. Trends Genet 2010, 26: $21-28$.

5. Marek MS, Johnson-Buck A, Walter NG. The shapeshifting quasispecies of RNA: one sequence, many functional folds. Phys Chem Chem Phys 2011, 13:11524-11537.

6. Frank J, Gonzalez RL Jr. Structure and dynamics of a processive Brownian motor: the translating ribosome. Annu Rev Biochem 2010, 79: $381-412$.

7. Will CL, Luhrmann R. Spliceosome structure and function. Cold Spring Harb Perspect Biol 2011, 3:a003707.

8. Newman AJ, Nagai K. Structural studies of the spliceosome: blind men and an elephant. Curr Opin Struct Biol 2010, 20:82-89.

9. Mattick JS. The genetic signatures of noncoding RNAs. PLoS Genet 2009, 5:e1000459.

10. Seeman NC. Nanomaterials based on DNA. Annu Rev Biochem 2010, 79:65-87.

11. Rothemund PW. Folding DNA to create nanoscale shapes and patterns. Nature 2006, 440:297-302.

12. Seeman NC. An overview of structural DNA nanotechnology. Mol Biotechnol 2007, 37:246-257.

13. Aldaye FA, Palmer AL, Sleiman HF. Assembling materials with DNA as the guide. Science 2008, 321:1795-1799.

14. Nangreave J, Han D, Liu Y, Yan H. DNA origami: a history and current perspective. Curr Opin Chem Biol 2010, 14:608-615.

15. Shih WM, Lin C. Knitting complex weaves with DNA origami. Curr Opin Struct Biol 2010, 20:276-282.

16. Torring T, Voigt NV, Nangreave J, Yan H, Gothelf KV. DNA origami: a quantum leap for self-assembly of complex structures. Chem Soc Rev. In press.

17. Watson JD, Crick FH. Molecular structure of nucleic acids; a structure for deoxyribose nucleic acid. Nature 1953, 171:737-738.

18. Williams NH, Takasaki B, Wall M, Chin J. Structure and nuclease activity of simple dinuclear metal complexes: quantitative dissection of the role of metal ions. Acc Chem Res 1999, 32:485-493.

19. Wang S, Kool ET. Origins of the large differences in stability of DNA and RNA helices: C-5 methyl and 2'hydroxyl effects. Biochemistry 1995, 34:4125-4132.

20. Lipps HJ, Rhodes D. G-quadruplex structures: in vivo evidence and function. Trends Cell Biol 2009, 19:414-422.

21. Grindley ND, Whiteson KL, Rice PA. Mechanisms of site-specific recombination. Annu Rev Biochem 2006, 75:567-605

22. Schones DE, Zhao K. Genome-wide approaches to studying chromatin modifications. Nat Rev Genet 2008, 9:179-191.

23. Strahl BD, Allis CD. The language of covalent histone modifications. Nature 2000, 403:41-45.

24. Chen J, Darst SA, Thirumalai D. Promoter melting triggered by bacterial RNA polymerase occurs in three steps. Proc Natl Acad Sci U S A 2010, 107:12523-12528.

25. Crick F. Central dogma of molecular biology. Nature 1970, 227:561-563.

26. Davidson BL, McCray PB Jr. Current prospects for RNA interference-based therapies. Nat Rev Genet 2011, 12:329-340.

27. Al-Hashimi HM, Walter NG. RNA dynamics: it is about time. Curr Opin Struct Biol 2008, 18:321-329.

28. Dambach MD, Winkler WC. Expanding roles for metabolite-sensing regulatory RNAs. Curr Opin Microbiol 2009, 12:161-169. 
29. Nissen P, Hansen J, Ban N, Moore PB, Steitz TA. The structural basis of ribosome activity in peptide bond synthesis. Science 2000, 289:920-930.

30. Ferre-D’Amare AR, Scott WG. Small self-cleaving ribozymes. Cold Spring Harb Perspect Biol 2010, 2:a003574.

31. Ehrlich M, Gama-Sosa MA, Carreira LH, Ljungdahl LG, Kuo KC, Gehrke CW. DNA methylation in thermophilic bacteria: N4-methylcytosine, 5methylcytosine, and N6-methyladenine. Nucleic Acids Res 1985, 13:1399-1412.

32. Jones PA, Takai D. The role of DNA methylation in mammalian epigenetics. Science 2001, 293:1068-1070.

33. Campbell MA, Wengel J. Locked vs. unlocked nucleic acids (LNA vs. UNA): contrasting structures work towards common therapeutic goals. Chem Soc Rev 2011.

34. Braasch DA, Corey DR. Locked nucleic acid (LNA): fine-tuning the recognition of DNA and RNA. Chem Biol 2001, 8:1-7.

35. Rana TM. Illuminating the silence: understanding the structure and function of small RNAs. Nat Rev Mol Cell Biol 2007, 8:23-36.

36. Egholm M, Buchardt O, Christensen L, Behrens C, Freier SM, Driver DA, Berg RH, Kim SK, Norden B, Nielsen PE. PNA hybridizes to complementary oligonucleotides obeying the Watson-Crick hydrogenbonding rules. Nature 1993, 365:566-568.

37. Williams KA, Veenhuizen PT, de la Torre BG, Eritja R, Dekker C. Nanotechnology: carbon nanotubes with DNA recognition. Nature 2002, 420:761.

38. Lukeman PS, Mittal AC, Seeman NC. Two dimensional PNA/DNA arrays: estimating the helicity of unusual nucleic acid polymers. Chem Commun 2004, 1694-1695.

39. Chen F, Yang Z, Yan M, Alvarado JB, Wang G, Benner SA. Recognition of an expanded genetic alphabet by type-II restriction endonucleases and their application to analyze polymerase fidelity. Nucleic Acids Res 2011, 39:3949-3961.

40. Petrillo ML, Newton CJ, Cunningham RP, Ma RI, Kallenbach NR, Seeman NC. The ligation and flexibility of 4-arm DNA junctions. Biopolymers 1988, 27:1337-1352.

41. Holliday R. Mechanism for gene conversion in fungi. Genet Res 1964, 5:282-304.

42. Fu TJ, Seeman NC. DNA double-crossover molecules. Biochemistry 1993, 32:3211-3220.

43. LaBean TH, Yan H, Kopatsch J, Liu FR, Winfree E, Reif JH, Seeman NC. Construction, analysis, ligation, and self-assembly of DNA triple crossover complexes. J Am Chem Soc 2000, 122:1848-1860.

44. Shih WM, Quispe JD, Joyce GF. A 1.7-kilobase singlestranded DNA that folds into a nanoscale octahedron. Nature 2004, 427:618-621.
45. Han D, Pal S, Nangreave J, Deng Z, Liu Y, Yan H. DNA origami with complex curvatures in threedimensional space. Science 2011, 332:342-346.

46. Woo S, Rothemund PW. Programmable molecular recognition based on the geometry of DNA nanostructures. Nat Chem 2011, 3:620-627.

47. Lund K, Manzo AJ, Dabby N, Michelotti N, JohnsonBuck A, Nangreave J, Taylor S, Pei R, Stojanovic MN, Walter NG, et al. Molecular robots guided by prescriptive landscapes. Nature 2010, 465:206-210.

48. Gu HZ, Chao J, Xiao SJ, Seeman NC. A proximitybased programmable DNA nanoscale assembly line. Nature 2010, 465:202-286.

49. He Y, Liu DR. Autonomous multistep organic synthesis in a single isothermal solution mediated by a DNA walker. Nat Nanotechnol 2010, 5:778-782.

50. Maune HT, Han SP, Barish RD, Bockrath M, Goddard WA, Rothemund PWK, Winfree E. Self-assembly of carbon nanotubes into two-dimensional geometries using DNA origami templates. Nat Nanotechnol 2010, $5: 61-66$

51. Eskelinen AP, Kuzyk A, Kaltiaisenaho TK, Timmermans MY, Nasibulin AG, Kauppinen EI, Torma P. Assembly of single-walled carbon nanotubes on DNAorigami templates through streptavidin-biotin interaction. Small 2011, 7:746-750.

52. Michelotti N, de Silva C, Johnson-Buck AE, Manzo AJ, Walter NG. A bird's eye view tracking slow nanometer-scale movements of single molecular nanoassemblies. Methods Enzymol 2010, 475:121-148.

53. von Delius M, Leigh DA. Walking molecules. Chem Soc Rev 2011, 40:3656-3676.

54. Qian L, Winfree E. Scaling up digital circuit computation with DNA strand displacement cascades. Science 2011, 332:1196-1201.

55. Boeneman K, Prasuhn DE, Blanco-Canosa JB, Dawson PE, Melinger JS, Ancona M, Stewart MH, Susumu K, Huston A, Medintz IL. Self-assembled quantum dot-sensitized multivalent DNA photonic wires. I Am Chem Soc 2010, 132:18177-18190.

56. Hung AM, Micheel CM, Bozano LD, Osterbur LW, Wallraff GM, Cha JN. Large-area spatially ordered arrays of gold nanoparticles directed by lithographically confined DNA origami. Nat Nanotechnol 2010, 5:121-126.

57. Lin CX, Ke YG, Liu Y, Mertig M, Gu J, Yan H. Functional DNA nanotube arrays: bottom-up meets top-down. Angew Chem Int Ed Engl 2007, 46:6089-6092.

58. Niemeyer CM, Koehler J, Wuerdemann C. DNAdirected assembly of bienzymic complexes from in vivo biotinylated $\mathrm{NAD}(\mathrm{P}) \mathrm{H}$ : FMN oxidoreductase and luciferase. Chembiochem 2002, 3:242-245.

59. Ruiz-Carretero A, Janssen PGA, Stevens AL, Surin M, Herz LM, Schenning APHJ. Directing energy transfer 
in discrete one-dimensional oligonucleotide-templated assemblies. Chem Commun 2011, 47:884-886.

60. Stein IH, Steinhauer C, Tinnefeld P. Single-molecule four-color FRET visualizes energy-transfer paths on DNA origami. J Am Chem Soc 2011, 133:4193-4195.

61. Singh A, Tolev M, Meng M, Klenin K, Plietzsch O, Schilling CI, Muller T, Nieger M, Brase S, Wenzel W, et al. Branched DNA that forms a solid at 95 degrees C. Angew Chem Int Ed Engl 2011, 50:3227-3231.

62. Kwak M, Herrmann A. Nucleic acid/organic polymer hybrid materials: synthesis, superstructures, and applications. Angew Chem Int Ed Engl 2010, 49:8574-8587.

63. Ohno H, Kobayashi T, Kabata R, Endo K, Iwasa T, Yoshimura SH, Takeyasu K, Inoue T, Saito H. Synthetic RNA-protein complex shaped like an equilateral triangle. Nat Nanotechnol 2011, 6:115-119.

64. Hung AM, Noh H, Cha JN. Recent advances in DNAbased directed assembly on surfaces. Nanoscale 2010, 2:2530-2537.

65. Kershner RJ, Bozano LD, Micheel CM, Hung AM, Fornof AR, Cha JN, Rettner CT, Bersani M, Frommer J, Rothemund PW, et al. Placement and orientation of individual DNA shapes on lithographically patterned surfaces. Nat Nanotechnol 2009, 4:557-561.

66. Stojanovic MN, Mitchell TE, Stefanovic D. Deoxyribozyme-based logic gates. J Am Chem Soc 2002, 124:3555-3561.

67. Stojanovic MN, Stefanovic D. A deoxyribozymebased molecular automaton. Nat Biotechnol 2003, 21:1069-1074.

68. Pei R, Matamoros E, Liu M, Stefanovic D, Stojanovic MN. Training a molecular automaton to play a game. Nat Nanotechnol 2010, 5:773-777.

69. Qian L, Winfree E, Bruck J. Neural network computation with DNA strand displacement cascades. Nature 2011, 475:368-372.

70. Morris MK, Saez-Rodriguez J, Sorger PK, Lauffenburger DA. Logic-based models for the analysis of cell signaling networks. Biochemistry 2010, 49:3216-3224.

71. Ozbay E. Plasmonics: merging photonics and electronics at nanoscale dimensions. Science 2006, 311:189-193.

72. Anker JN, Hall WP, Lyandres O, Shah NC, Zhao J, Van Duyne RP. Biosensing with plasmonic nanosensors. Nat Mater 2008, 7:442-453.

73. Tan SJ, Campolongo MJ, Luo D, Cheng W. Building plasmonic nanostructures with DNA. Nat Nanotechnol 2011, 6:268-276.

74. Ding B, Deng Z, Yan H, Cabrini S, Zuckermann RN, Bokor J. Gold nanoparticle self-similar chain structure organized by DNA origami. J Am Chem Soc 2010, 132:3248-3249.
75. Lo PK, Karam P, Aldaye FA, McLaughlin CK, Hamblin GD, Cosa G, Sleiman HF. Loading and selective release of cargo in DNA nanotubes with longitudinal variation. Nat Chem 2010, 2:319-328.

76. Rothemund PW, Ekani-Nkodo A, Papadakis N, Kumar A, Fygenson DK, Winfree E. Design and characterization of programmable DNA nanotubes. $J \mathrm{Am}$ Chem Soc 2004, 126:16344-16352.

77. Palchetti I, Mascini M. Nucleic acid biosensors for environmental pollution monitoring. Analyst 2008, 133:846-854.

78. Sekella PT, Rueda D, Walter NG. A biosensor for theophylline based on fluorescence detection of ligandinduced hammerhead ribozyme cleavage. RNA 2002, $8: 1242-1252$.

79. Modi S, Swetha MG, Goswami D, Gupta GD, Mayor S, Krishnan Y. A DNA nanomachine that maps spatial and temporal $\mathrm{pH}$ changes inside living cells. Nat Nanotechnol 2009, 4:325-330.

80. Wang J. DNA biosensors based on peptide nucleic acid (PNA) recognition layers. A review. Biosens Bioelectron 1998, 13:757-762.

81. Martinez K, Estevez MC, Wu Y, Phillips JA, Medley CD, Tan W. Locked nucleic acid based beacons for surface interaction studies and biosensor development. Anal Chem 2009, 81:3448-3454.

82. Piunno PA, Krull UJ. Trends in the development of nucleic acid biosensors for medical diagnostics. Anal Bioanal Chem 2005, 381:1004-1011.

83. Ke Y, Lindsay S, Chang Y, Liu Y, Yan H. Selfassembled water-soluble nucleic acid probe tiles for label-free RNA hybridization assays. Science 2008, 319:180-183.

84. Liu HJ, Torring T, Dong MD, Rosen CB, Besenbacher F, Gothelf KV. DNA-templated covalent coupling of G4 PAMAM dendrimers. J Am Chem Soc 2010, 132:18054-18056.

85. Crawford JM, Townsend CA. New insights into the formation of fungal aromatic polyketides. Nat Rev Microbiol 2010, 8:879-889.

86. Andrianantoandro E, Basu S, Karig DK, Weiss R. Synthetic biology: new engineering rules for an emerging discipline. Mol Syst Biol 2006, 2:2006.0028.

87. Lee SK, Chou H, Ham TS, Lee TS, Keasling JD. Metabolic engineering of microorganisms for biofuels production: from bugs to synthetic biology to fuels. Curr Opin Biotechnol 2008, 19:556-563.

88. Gibson DG, Glass JI, Lartigue C, Noskov VN, Chuang RY, Algire MA, Benders GA, Montague MG, Ma L, Moodie MM, et al. Creation of a bacterial cell controlled by a chemically synthesized genome. Science 2010, 329:52-56.

89. Wilner OI, Shimron S, Weizmann Y, Wang ZG, Willner I. Self-assembly of enzymes on DNA scaffolds: en route to biocatalytic cascades and the synthesis of metallic nanowires. Nano Lett 2009, 9:2040-2043. 
90. Andersen ES, Dong M, Nielsen MM, Jahn K, Subramani R, Mamdouh W, Golas MM, Sander B, Stark H, Oliveira CL, et al. Self-assembly of a nanoscale DNA box with a controllable lid. Nature 2009, 459:73-76.

91. Endo M, Hidaka K, Kato T, Namba K, Sugiyama H. DNA prism structures constructed by folding of multiple rectangular arms. J Am Chem Soc 2009, 131:15570-15571.

92. Venkataraman S, Dirks RM, Ueda CT, Pierce NA. Selective cell death mediated by small conditional RNAs. Proc Natl Acad Sci U S A 2010, 107:16777-16782.

93. Surana S, Bhat JM, Koushika SP, Krishnan Y. An autonomous DNA nanomachine maps spatiotemporal $\mathrm{pH}$ changes in a multicellular living organism. Nat Commun 2011, 2:340.

94. Bhatia D, Surana S, Chakraborty S, Koushika SP, Krishnan Y. A synthetic icosahedral DNA-based hostcargo complex for functional in vivo imaging. Nat Commun 2011, 2:339.

95. Mei Q, Wei X, Su F, Liu Y, Youngbull C, Johnson R, Lindsay S, Yan H, Meldrum D. Stability of DNA origami nanoarrays in cell lysate. Nano Lett 2011, 11:1477-1482.

96. Lu W, Lieber CM. Nanoelectronics from the bottom up. Nat Mater 2007, 6:841-850.

97. Engheta N. Circuits with light at nanoscales: optical nanocircuits inspired by metamaterials. Science 2007, 317:1698-1702.
98. Tour JM, Kittrell C, Colvin VL. Green carbon as a bridge to renewable energy. Nat Mater 2010, 9:871-874.

99. Solomon S, Plattner GK, Knutti R, Friedlingstein P. Irreversible climate change due to carbon dioxide emissions. Proc Natl Acad Sci U S A 2009, 106:1704-1709.

100. Delebecque CJ, Lindner AB, Silver PA, Aldaye FA. Organization of intracellular reactions with rationally designed RNA assemblies. Science 2011, 333:470-474.

101. Moghimi SM, Hunter AC, Murray JC. Nanomedicine: current status and future prospects. FASEB J 2005, 19:311-330.

102. Brandenburg B, Lee LY, Lakadamyali M, Rust MJ, Zhuang X, Hogle JM. Imaging poliovirus entry in live cells. PLoS Biol 2007, 5:e183.

103. Benenson Y, Gil B, Ben-Dor U, Adar R, Shapiro E. An autonomous molecular computer for logical control of gene expression. Nature 2004, 429:423-429.

104. Krishna H, Caruthers MH. Solid-phase synthesis, thermal denaturation studies, nuclease resistance, and cellular uptake of (oligodeoxyribonucleoside)methylborane phosphine-DNA chimeras. I Am Chem Soc 2011, 133:9844-9854.

105. Rueda D, Walter NG. Single molecule fluorescence control for nanotechnology. J Nanosci Nanotechnol 2005, 5:1990-2000.

106. Walter NG, Huang CY, Manzo AJ, Sobhy MA. Do-ityourself guide: how to use the modern single-molecule toolkit. Nat Methods 2008, 5:475-489.

\section{FURTHER READING}

von Delius M, Leigh D. Walking molecules. Chem Soc Rev 2011, 40:3656-3676.

Faisal A, Alison P, Hanadi S. Assembling materials with DNA as the guide. Science 2008, 321:1795-1799

http://en.wikipedia.org/wiki/DNA_nanotechnology

http://en.wikipedia.org/wiki/Fuzzy_logic

http://en.wikipedia.org/wiki/Nanoelectronics

http://en.wikipedia.org/wiki/Nanomedicine

$\mathrm{Hu}$ Y, Fine DH, Tasciotti E, Bouamrani A, Ferrari M. Nanodevices in diagnostics. WIREs Nanomed Nanobiotechnol 2011, 3:11-32.

Nadrian S. DNA in a material world. Nature 2003, 421:427-431.

Nadrian S. Nanomaterials based on DNA. Annu Rev Biochem 2010, 79:65-87.

Rothemund PW. Folding DNA to create nanoscale shapes and patterns. Nature 2006, 440:297-302.

Yang D, Campolongo MJ, Nhi Tran TN, Ruiz RC, Kahn JS, Luo D. Novel DNA materials and their applications. WIREs Nanomed Nanobiotechnol 2010, 2:648-669. 